\title{
64-fs L-band Pulse Generation by an All-Fibre Er-Doped Laser
}

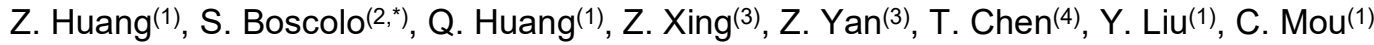 \\ (1) Key Laboratory of Specialty Fiber Optics and Optical Access Network, Shanghai University, \\ Shanghai 200444, China \\ (2) Aston Institute of Photonic Technologies, Aston University, Birmingham B4 7ET, United Kingdom \\ (3) National Engineering Laboratory for Next Generation Internet Access System, Huazhong University \\ of Science and Technology, Wuhan 430074, China \\ (4) Key Laboratory of Space Active Opto-Electronics Technology, Shanghai Institute of Technical \\ Physics, Shanghai 200083, China \\ s.a.boscolo@aston.ac.uk
}

\begin{abstract}
We demonstrate a L-band all-fibre erbium-doped laser mode-locked by nonlinear polarisation rotation. The use of a single gain segment with appropriate length and dispersion and a L-band optimised Brewster fibre grating as an in-fibre polariser enables the generation of 64-fs pulses at 1.59 $\mu m$.
\end{abstract}

\section{Introduction}

Femtosecond fibre oscillators offer significant advantages over solid-state systems because of their simplicity, cost, and reliability. Ultrashort erbium-doped fibre lasers (EDFLs) working in the $1.6 \mu \mathrm{m}$ band have attracted much attention in recent years, owing to their capability to expand the band of telecommunications and sensing ${ }^{[1],[2],}$ and their wide applications in spectroscopy, biomedical diagnostics and surgery. Operation of EDFLs at $1.6 \mu \mathrm{m}$ can be realised through control of the linear cavity loss ${ }^{[3]}$ and based on this principle, a number of studies on L-band EDFLs mode-locked through nonlinear polarisation rotation (NPR) or saturable absorbers have been reported $[4],[5]$. However, these lasers operate in the soliton regime and, thus, the pulse duration and energy are constrained. Whilst lengthening the EDF can force the laser emit in the $L$ band[1],[6],[7], the extra losses introduced by an overlong EDF make it difficult for the laser to achieve stable mode locking. Moreover, in lasers operating at net normal dispersion, large bandwidths and high pulse energies are favoured by short fibre cavities. Sub-100-fs $L$-band pulses can be obtained from fibre lasers exploiting the similariton ${ }^{[8]}$ or dissipative-soliton ${ }^{[9]}$ pulse formation mechanisms ${ }^{[10,11]}$. However, such laser designs require two segments of different types of EDFs to force the laser operate at $1.6 \mu \mathrm{m}$, hence two pump diodes.

In this paper, we demonstrate the generation of 64-fs stretched pulses ${ }^{[12]}$ from an all-fibre erbiumdoped laser emitting in the $1.6-\mu \mathrm{m}$ band, which uses a Brewster fibre grating optimised for the $L$ band as an in-fbre polariser ${ }^{[13]}$ and only one section of EDF with appropriate length and dispersion. The design is simple and suitable for all-fibre integration.

\section{Laser configuration and principle}
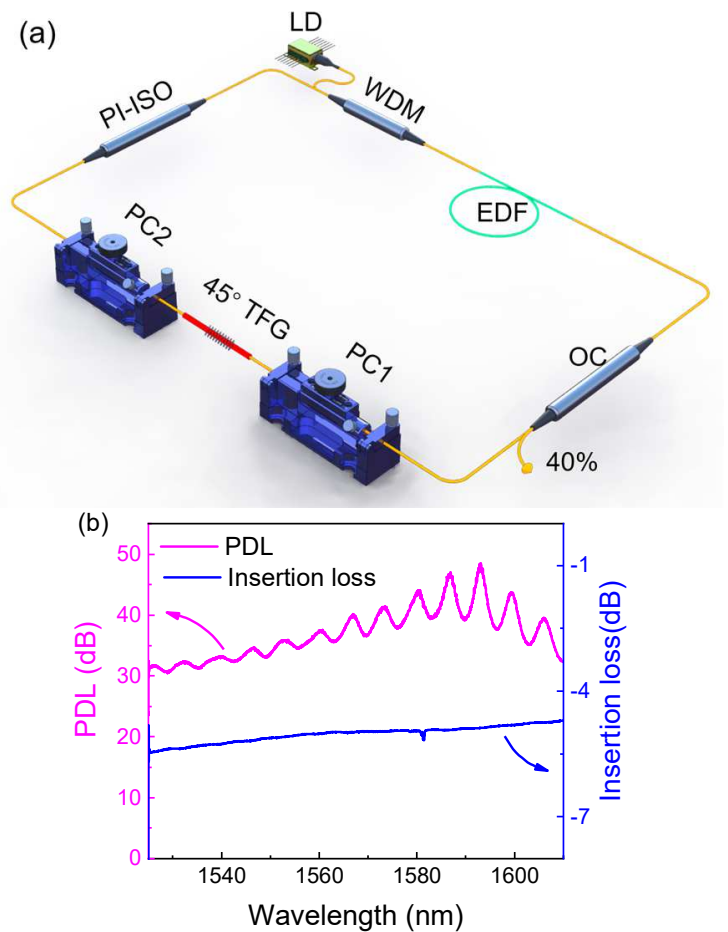

Fig. 1: (a) Experimental setup of the $L$-band stretched-pulse mode-locked fibre laser. (b) PDL response and insertion loss of the Brewster fibre grating.

The experimental setup is sketched in Fig. 1(a). A $1.575-\mathrm{m}$-long highly doped EDF with a nominal absorption coefficient of $\sim 80 \mathrm{~dB} / \mathrm{m}$ at $1530 \mathrm{~nm}$ and a group velocity dispersion (GVD) of -45.1 $\mathrm{ps} /(\mathrm{nm} \cdot \mathrm{km})$ at $1590 \mathrm{~nm}$ constitutes the gain medium. The EDF is pumped by a laser diode operating at $980 \mathrm{~nm}$ through a wavelengthdivision multiplexer and providing up to 704-mW pump power. Other fibres in the cavity are standard single-mode fibre (SMF) with a GVD of $+19.4 \mathrm{ps} /(\mathrm{nm} \cdot \mathrm{km})$ at $1590 \mathrm{~nm}$, yielding a small normal cavity dispersion, which triggers 
operation of the laser in the stretched-pulse regime ${ }^{[12]}$. A Brewster fibre grating with strong polarisation-dependent loss (PDL) sandwiched with two polarisation controllers, converts NPR to amplitude modulation, initiating and stabilising mode-locked operation ${ }^{[14,15]}$. A polarisationindependent isolator ensures single direction oscillation. A fibre coupler after the EDF taps $40 \%$ of laser power out of the cavity for measurement. In our laser setup, the relatively long EDF length permits to acquire $1.6 \mu \mathrm{m}$ emission, while the strong PDL of the Brewster fibre grating favours the generation of ultrashort pulses ${ }^{[16]}$. The Brewster fibre grating is UV inscribed into a length of hydrogenated SMF using the standard phase mask scanning technique. A description of its operation principle and fabrication procedure can be found in Ref.[17]. Figure 1(b) shows the transmission properties of the grating over the spectral range $1525 \mathrm{~nm}$ to $1610 \mathrm{~nm}$, acquired by a commercial optical vector analyser incorporating a tunable laser. It is seen that the grating features a PDL (measured as the peakto-peak difference in transmission with respect to all possible states of polarisation) of $\sim 48 \mathrm{~dB}$ at $1593 \mathrm{~nm}$, while the PDL remains well above 35 $\mathrm{dB}$ over a wide range of the $L$-band.

\section{Results}

Through management of the cavity dispersion realised by finely tuning the SMF length, we found that the optimal cavity length is $5.02 \mathrm{~m}$, yielding a net dispersion of $\sim 0.006 \mathrm{ps}^{2}$ at 1590 $\mathrm{nm}$. Under this cavity length, mode-locked operation of the laser can be easily obtained when the pump power is above $150 \mathrm{~mW}$ by properly adjusting the PCs. The performance of the laser at 200-mW pump power is summarised in Fig. 2. The output pulse train as observed on the oscilloscope (Fig. 2(a)) shows a pulse spacing of $\sim 24.6 \mathrm{~ns}$, yielding a repetition rate of $\sim 40.63 \mathrm{MHz}$. The signal-to-noise ratio in the radio-frequency spectrum of the laser output (Fig. 2(b)), measured by a RF spectrometer with a resolution bandwidth of $1 \mathrm{kHz}$ over a $1-\mathrm{MHz}$ range, is $58.4 \mathrm{~dB}$. The low noise background in the RF spectrum over a $3.2-\mathrm{GHz}$ range $(10-\mathrm{kHz}$ resolution bandwidth) also provides evidence of stable single-pulse mode-locking operation. The corresponding Kelly sideband-free, wide optical spectrum profile shown in Fig. 2(c) is a signature of the stretched-pulse operation regime. The spectrum, measured with a $0.05-\mathrm{nm}$ resolution, is centred on $1591 \mathrm{~nm}$ and has a bandwidth at full width at half-maximum (FWHM) of $65.1 \mathrm{~nm}$. The autocorrelation trace of the pulse (Fig. 2(d)) indicates a FWHM pulse duration of 240 fs when a Gaussian fit is assumed. By optimising the SMF pigtail of the laser output port, the pulses can be de-chirped to $64 \mathrm{fs}$. The time-bandwidth product of the compressed pulses is 0.49 , which is very close to the Fourier transform limit for Gaussian pulses (0.441). These pulses are the shortest pulses generated so far in the $L$-band from a fibre laser using a single segment of EDF. The average output power is $16.5 \mathrm{~mW}$, corresponding to a pulse energy of $0.41 \mathrm{~nJ}$. Increasing the pump power beyond $200 \mathrm{~mW}$ resulted in multiple pulse formation in the laser as a result of a peak-powerlimiting effect of the laser cavity[18].
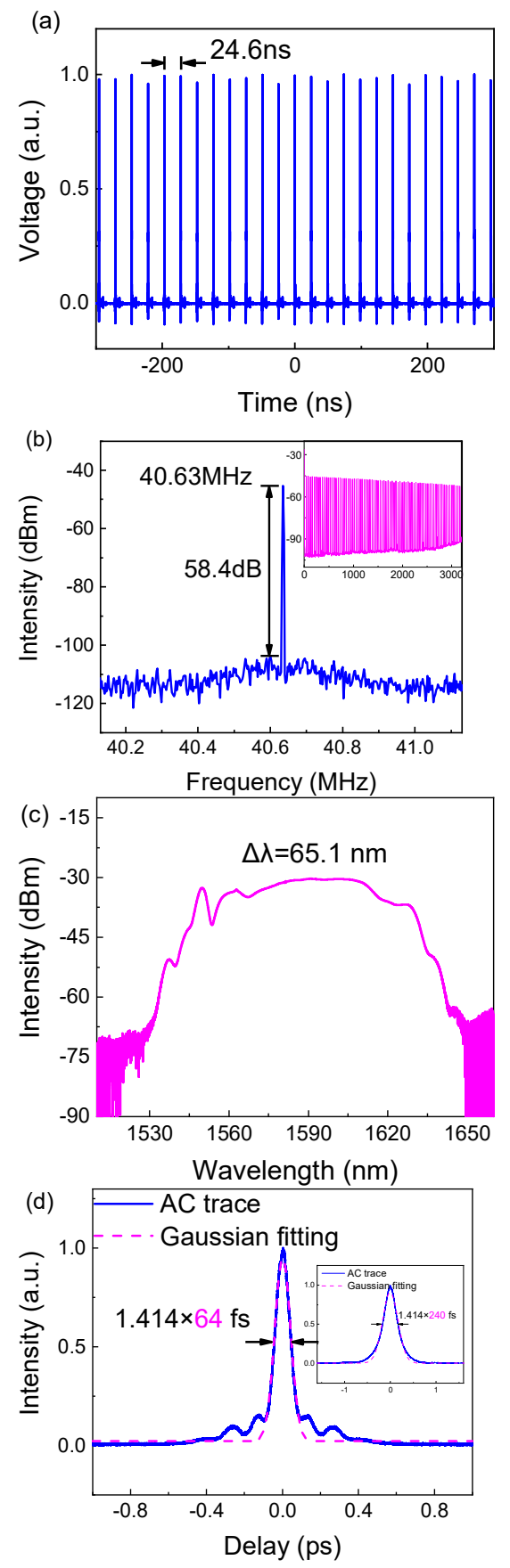

Fig. 2: Mode-locking operation at 200-mW pump power. (a) Pulse train. (b) RF spectrum. (c) Optical spectrum. (d) Autocorrelation traces of the chirped and dechirped (inset) pulses. 
To confirm the pulse generation regime in the laser, we performed numerical simulations of the laser based on a non-distributed model that includes the dominant physical effects of the system, namely GVD, self-phase modulation, gain saturation and bandwidth-limited gain for the EDF, as well as the discrete effects of the saturable absorber element. The simulation parameters were the same as the experimental values. The spectral width at FWHM of the simulated output pulses and the FWHM pulse duration after de-chirping were $63.1 \mathrm{~nm}$ and 60 fs, respectively, in a good agreement with the measured values. The in-cavity pulse evolution displayed in Fig. 3 clearly shows that the laser operates in the stretched-pulse regime ${ }^{[12]}$ : the pulse temporally stretches and compresses twice per round-trip, reaches a minimum duration in the middle of the EDF and SMF segments, and acquires both signs of chirp.

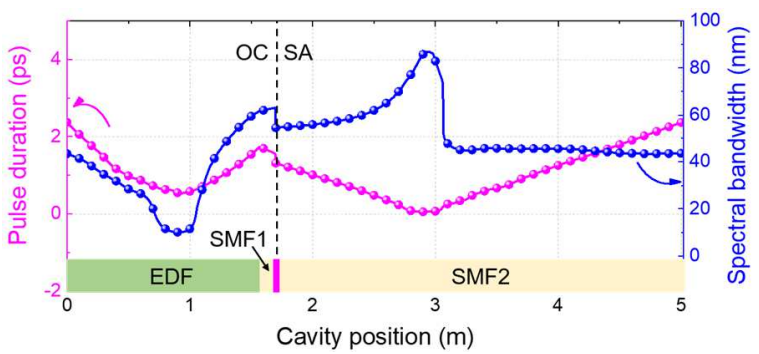

Fig. 3: Simulated evolution of the FWHM temporal (red) and spectral (blue) widths of the pulse along the cavity.

\section{Conclusions}

We demonstrated a sub-70-fs all-fibre erbiumdoped laser that operates in the L-band and requires only one gain segment. This laser delivers pulses at $40.63-\mathrm{MHz}$ repetition rate and 1.59- $\mu \mathrm{m}$ central wavelength that can be compressed externally to 64 fs. To our knowledge, this is the shortest duration obtained in L-band EDFLs using a single gain segment. Our laser design lends itself well to fibre integration, and thus will be highly desirable for a variety of applications requiring $L$-band ultrashort pulses.

\section{Acknowledgements}

This research was supported by the National Natural Science Foundation of China (61975107), the Natural Science Foundation of Shanghai (20ZR1471500), and the UK EPSRC (EP/S003436/1 - PHOS).

\section{References}

[1] X. Dong et al., "Effects of active fiber length on the tunability of erbium-doped fiber ring lasers," Opt. Express, vol. 11, pp. 3622-3627, 2003.

[2] S. M. Fernandez-Vallejo, D. Ardanaz, and M. LópezAmo, "Optimization of the available spectrum of a WDM sensors network using a mode-locked laser," J. Lightwave. Technol., vol. 33, no. 22, pp. 4627-4631, 2015.
[3] K. Guesmi et al., "1.6 $\mu \mathrm{m}$ emission based on linear loss control in a Er:Yb doped double-clad fiber laser," Opt. Lett., vol. 39, pp. 6383-6386, 2014.

[4] Y. Meng et al., "Mode-locked Er:Yb-doped double-clad fiber laser with 75-nm tuning range," Opt. Lett., vol. 40, pp. 1153-1156, 2015.

[5] Y. Meng et al., "1.61 $\mu \mathrm{m}$ high-order passive harmonic mode locking in a fiber laser based on graphene saturable absorber," Opt. Express, vol. 22, pp. 2992129926, 2014.

[6] M. Melo et al., "Tunable L-band erbium-doped fibre ring laser by means of induced cavity loss using a fibre taper," Appl. Phys. B, vol. 77, pp. 139-142, 2003.

[7] D. Yan et al., "L-band wavelength-tunable dissipative soliton fiber laser," Opt. Express, vol. 24, pp. 739-748, 2016.

[8] F.Ö. Ilday et al., "Self-similar evolution of parabolic pulses in a laser," Phys. Rev. Lett., vol. 92, 213902(4), 2004.

[9] A. Chong, J. Buckley, W. Renninger, and F. Wise, "Allnormal-dispersion femtosecond fiber laser," Opt. Express, vol. 14, pp. 10095-10100, 2006.

[10] Z. Wang et al., "Generation of sub-60 fs similaritons at $1.6 \mu \mathrm{m}$ from an all-fiber Er-doped laser," J. Lightwave Technol., vol. 34, pp. 4127-4133, 2016.

[11] Z. Wang et al., "Sub-90 fs dissipative-soliton erbiumdoped fiber lasers operating at $1.6 \mu \mathrm{m}$ band," Opt. Express, vol. 24, pp. 10841-10846, 2016.

[12] K. Tamura, E. P. Ippen, H. A. Haus, and L. E. Nelson, "77-fs pulse generation from a stretched-pulse modelocked all-fiber ring laser," Opt. Lett., vol. 18, pp. 10801082, 1993.

[13] K. Zhou et al., "High extinction ratio in-fiber polarizers based on $45^{\circ}$ tilted fiber Bragg gratings," Opt. Lett., vol. 30, pp. 1285-1287, 2005.

[14] C. Mou et al., "All-fiber passively mode-locked femtosecond laser using a $45^{\circ}$-tilted fiber grating polarization element," Opt. Express, vol. 18, pp. 18906-18911, 2010.

[15] Z. Zhang et al., "Sub-100 fs mode-locked erbiumdoped fiber laser using a $45^{\circ}$-tilted fiber grating," Opt. Express, vol. 21, pp. 28297-28303, 2013.

[16] T. Wang et al., "Mode-locked erbium-doped fiber lasers using $45^{\circ}$ tilted fiber grating," IEEE J. Sel. Top. Quantum Electron., vol. 24, pp. 1-6, 2018.

[17] Z. Yan et al., "UV-inscription, polarization-dependant loss characteristics and applications of $45^{\circ}$ tilted fiber gratings," J. Lightwave Technol., vol. 29, pp. 2715$2724,2011$.

[18] D. Y. Tang, L. M. Zhao, B. Zhao, and A. Q. Liu, "Mechanism of multisoliton formation and soliton energy quantization in passively mode-locked fiber lasers," Phys. Rev. A, vol. 72, 043816(9), 2005. 